Research Article

\title{
Metal-Assisted and Microwave-Accelerated Decrystallization
}

Yehnara S.B. Ettinoffe, Bridgit M. Kioko, Brittney I. Gordon, Nishone A. Thompson, Morenike Adebiyi, Kevin Mauge-Lewis, Taiwo O. Ogundolie, Enock Bonyi, Muzaffer Mohammed, Kadir Aslan

Morgan State University, Department of Chemistry, 1700 East Cold Spring Lane, Baltimore, MD 21251, USA

Corresponding author: E-mail: kadir.aslan@morgan.edu

Received: Oct. I, 2015; Accepted: Dec. 7, 2015; Published: Dec. 14, 2015.

Citation: Yehnara S.B. Ettinoffe, Bridgit M. Kioko, Brittney I. Gordon, Nishone A. Thompson, Morenike Adebiyi, Kevin Mauge-Lewis, Taiwo O. Ogundolie, Enock Bonyi, Muzaffer Mohammed and Kadir Aslan. Metal-Assisted and Microwave-Accelerated Decrystallization. Nano Biomed. Eng. 20I5, 7(4), I39-152.

DOI: $10.5101 / n b e . v 7 i 4 . p 139-152$.

\begin{abstract}
We present a platform technology, called Metal-Assisted and Microwave-Accelerated Decrystallization (MAMAD), which is based on the use of dispersion of gold colloids with low power microwave heating to decrystallize organic and biological crystals attached to surfaces. Uric acid crystals were chosen as model target crystals to be decrystallized using MAMAD technique. A two-step procedure was employed: 1) growth of uric acid crystals on a model surface (collagen films coated on to glass slides to simulate a human joint) at room temperature and 2) de-crystallization of uric acid crystals in synovial fluid (in vitro) using silver and gold colloids in conjunction with low power microwave heating. Using the MAMAD technique with gold colloids, the number of uric acid crystals was drastically reduced by $80 \%$ after $10 \mathrm{~min}$, where the average size of the uric acid crystals was reduced from $125 \mu \mathrm{m}$ to $50 \mu \mathrm{m}$. In control experiments and with silver colloids that aggregated from the solution, the size and number of uric crystals remained unchanged, indicating that the combined use of only metal colloids in solution and microwave heating is effective for the de-crystallization of uric acid crystals in biological media.
\end{abstract}

Keywords: Uric acid; Hyperuricaemia; Crystallization; Gold colloids; Silver colloids; Medical microwaves; Microwave-induced temperature gradients

\section{Introduction}

Uric acid is produced in the human body as a direct result of the catabolic processes involved in the digestion of foods rich in purines and is released into the blood serum by cells as they undergo apoptosis [1, 2]. L Under normal conditions uric acid is dissolved in the blood plasma, processed by the kidneys and discarded from the body by excretion in the form of urine. Hyperuraecemia occurs when the serum urate levels are elevated beyond the kidneys' capacity for waste processing [3]. In the state of hyperuricaemia, uric acid in the blood plasma having increased beyond a critical concentration $(>7.0 \mathrm{mg} / \mathrm{dL}$ in men and $>5.7 \mathrm{mg} / \mathrm{dL}$ in women) [4], may begin to crystallize resulting in the accumulation of crystalline deposits in the synovial fluid of the joint [5]. Subsequently large and sometimes hard masses called tophi are formed, indicative of the late stages of the crystal-induced arthropathy: gout or uric acid deposition disease [6]. This condition is estimated to affect $3.9 \%$ of the US adult population, or a reported 8.3 million individuals: 
a two-fold increase in the rate of incidence over a twenty-year period of study, with significantly higher rates of incidence among males, African-Americans and individuals above the age of 40 [7].

Numerous studies regarding possible treatments of the symptoms of gout have been reported in literature $[8,9]$. Previously, gout has been treated using alteration of suffers' dietary habits, application of ice, elevation and rest of the affected area and the administration of short-term medications including NSAIDs, colchicine and corticosteroids. Change in diet is specifically targeted to the long-term control of hyperuricaemia, while effective for the prevention of initial attack or relapses after treatment, there is little evidence to suggest that such a lifestyle change can be used as an effective treatment for individual attacks. Intensive pharmacological treatment of the disorder [10]. NSAIDs are commonly used for the treatment of arthritis but are linked to increased risk of the development of congestive heart failure [11, 12]. Olchicine, though used for the treatment of goutassociated pain during the early onset of an attack, is comparable in toxicity to arsenic and is blamed for muscle damage, neuropathy, and multiple organ failure $[13,14]$. Given the limitations of existing treatments of gout, it is important to find alternative methods of addressing the causative processes; the deposition of uric acid that resulting in progressive trauma to the affected joints.

Recently, the Aslan Research Group presented the initial observations for the use of colloidal gold solution with microwave heating for the decrystallization of uric acid crystals placed on unmodified glass slides, where $60 \%$ reduction in number of uric acid crystals were reported [15]. We hypothesized that the microwave-induced temperature gradients, which is generated between the metal colloids (not heated by microwaves) and the rest of the sample media (heated by microwaves), play an important role in the decrystallization of uric acid crystals by gold colloids. More specifically, the kinetic energy of the metal colloids in solution is increased by microwave heating, where the collisions between the metal colloids and uric acid crystals result in the break down uric acid crystals [15]. However, due to the preliminary nature of these observations reported in our initial paper [15], several questions, such as the use of modified surfaces to mimic the surface of a human bone, the effect of biological medium found in the joints of a human bone on the stability of metal colloids, the use of other metal colloids and temperature of the components of the system during microwave heating, were left unanswered.

In this work, we provide the results of our detailed investigation on the use of noble metal colloids in conjunction with low power microwave heating (i.e., Metal-Assisted and Microwave-Accelerated Decrystallization (MAMAD) technique, named for first time in this study) for the de-crystallization of uric acid crystals grown on collagen-modified glass slides in the presence of synovial fluid to demonstrate the potential use of MAMAD technique as an alternative treatment for gout. Gold colloids are used in the body for medical applications, particularly as tracers with applications to diagnostic medicine. The type and the concentration of gold colloids used in this study can be deemed to be non-toxic for human use based previous observations by others $[16,17]$.

Our results show that the use of MAMAD technique with gold colloids resulted in the significant reduction in the number and size of uric acid crystals. Substitution of silver colloids for gold colloids or the elimination of noble metal colloids altogether (control experiments) were both ineffective for the reduction of the number and size of uric acid crystals, and therefore deemed unsuitable for use in MAMAD technique. The temperature of the synovial fluid containing gold colloids and the collagen surface were increased from $23.5^{\circ} \mathrm{C}$ to $26.0^{\circ} \mathrm{C}$ and $26.5^{\circ} \mathrm{C}$ after 10 minutes of intermittent low power microwave heating, respectively. Optical absorption measurements of gold colloids in the presence of synovial fluid during intermittent microwave heating demonstrated that gold colloids remained in solution. Conversely, silver colloids were found to aggregate out of the solution, which provided evidence for the ineffectiveness of silver colloids in the reduction of significant reduction in the number and size of uric acid crystals.

\section{Materials and methods}

\section{Materials}

Uric acid, gold colloid (20 nm, optical density $=1)$ solution, hydrogen peroxide and sodium hydroxide (98\%) were purchased from Sigma-Aldrich. Dulbecco's Phosphate Buffered Saline (DPBS) (10X) and collagen I were purchased from Gibco Life Technologies. Sulfuric acid (reagent grade) and ethanol (200 proof/100\%) were obtained from Pharmco 
Products Inc. Hexyltrimethoxysilane (HTMS) was purchased from Gelest, Inc. Adhesive silicone isolators (2.0 $\mathrm{mm}$ in depth and $4.5 \mathrm{~mm}$ diameter) were obtained from Electron Microscopy Sciences (USA). Bovine synovial fluid was purchased from Lampire Biological Laboratories (USA). Single frosted glass microscope slides were obtained from Corning Inc. Bovine collagen, gold colloids (20 $\mathrm{nm}$ and of concentration $0.01 \%$, and were stored $4{ }^{\circ} \mathrm{C}$ and bovine synovial fluid at $-85{ }^{\circ} \mathrm{C}$ until ready for use. All other reagents were stored at room temperature and used as received except where otherwise stated.

\section{Methods}

Silanization of glass slides with HTMS. To simulate the de-crystallization of uric acid in biological medium in the joint of a bone, a glass slide was initially chemically etched using piranha solution (3:7 30\% hydrogen peroxide/concentrated sulfuric acid, followed by silanization by HTMS, which facilitates the binding of collagen for further surface modification as depicted in Scheme 1. HTMS is known to bind collagen through hydrophobic interactions.

HTMS solution was prepared using $47 \mathrm{~mL}$ of 200-proof ethanol, $2 \mathrm{~mL}$ of deionized water and 1 $\mathrm{mL}$ of HTMS in a $50 \mathrm{~mL}$ volumetric flask. The glass microscope slides were cut into several pieces (1 $\mathrm{cm} \times 1 \mathrm{~cm}$ in size), rinsed with deionized water and chemically etched by submersion in piranha solution for 10-15 minutes. After extensive washing, the slides were thoroughly rinsed with deionized water and subsequently dried by air. Cleaned slides were submerged in the freshly prepared HTMS solution and allowed to incubate at least for 30 minutes at $37^{\circ} \mathrm{C}$. The slides were washed thoroughly using ethanol and allowed to air-dry to remove all unbound materials. Silanized slides were then stored in an air-tight container for 24 hours at $37^{\circ} \mathrm{C}$ or for 30 minutes at $120^{\circ} \mathrm{C}$ before further modification.

\section{Preparation of collagen thin films}

Collagen thin films were prepared using previously

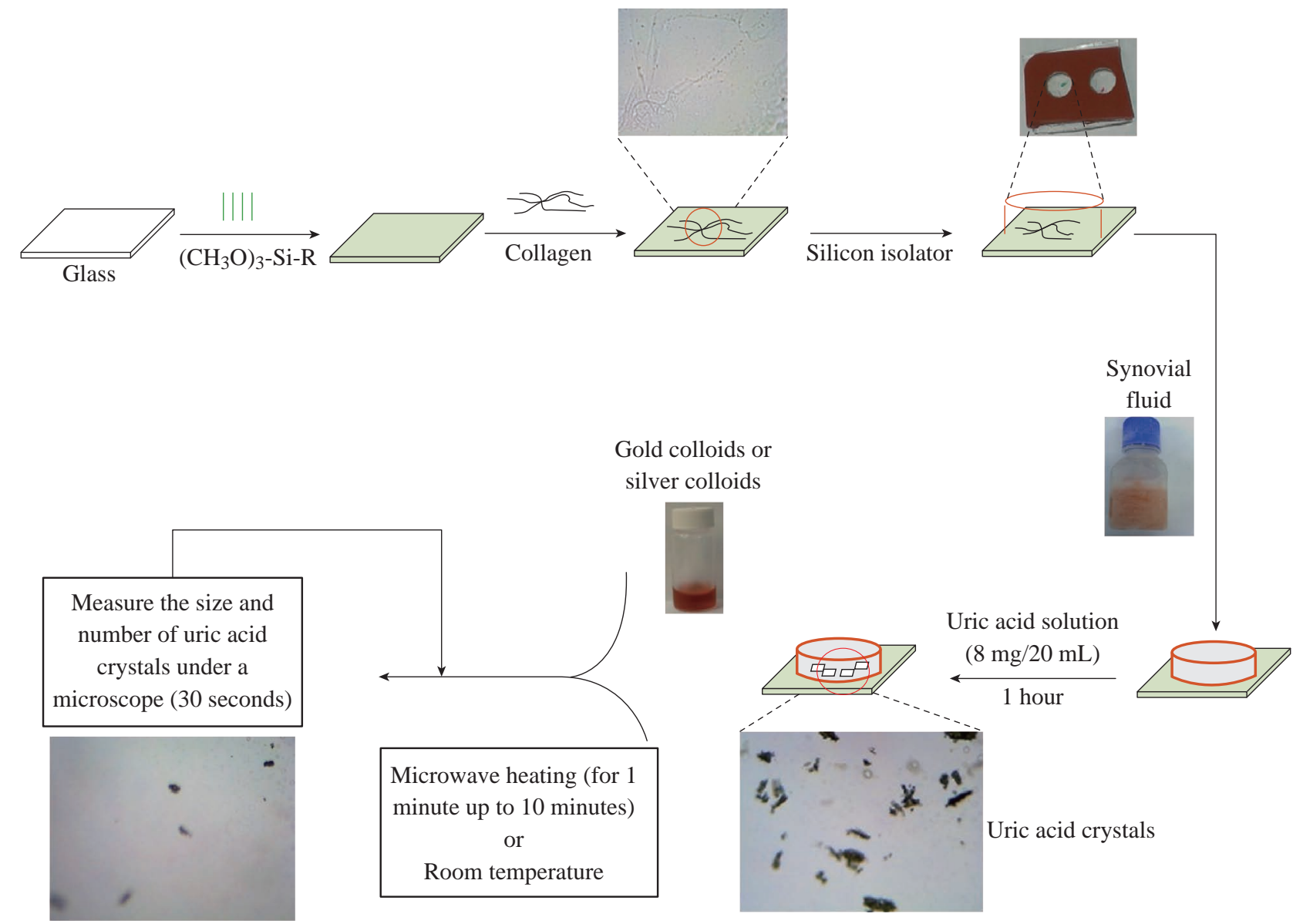

Scheme 1 Schematic depiction of the experiments. De-crystallization of uric acid crystals was investigated at room temperature (a control experiment) and using microwave heating (power level 1) with gold or silver colloids and in the absence of the colloids (a control experiment). 
established protocols $[18,19]$. All reagents were kept on ice under a sterile laminar flow hood, to prevent contamination or coagulation of the collagen. DPBS was diluted by a factor of 10 before use. $300 \mu \mathrm{L}$ of DPBS (1X), $4.8 \mu \mathrm{L}$ of $\mathrm{NaOH}$ and $192 \mu \mathrm{L}$ of collagen were combined in a $20 \mathrm{~mL}$ glass scintillation vial and gently swirled until thoroughly mixed. The glass slide pieces were placed in the collagen solution in an airtight container with the surface of interest facing upward. The slides were allowed to incubate at $37{ }^{\circ} \mathrm{C}$ for at least 16 hours prior to use. After incubation, glass slides were lifted out of the collagen solution allowing the gel to slide off gently. The collagen film was rinsed thoroughly with DPBS (1X) and deionized water. The collagen modified glass slides were air-dried and stored in DPBS (1X) at $4{ }^{\circ} \mathrm{C}$ until ready for use. Prior to the addition of any fluid to the surface-modified platform, silicon isolators were placed on the surface through the adhesive side to contain the liquid, delineate an area of focus and allow for the use of one surface for multiple experiments.

\section{Preparation of synovial fluid}

Synovial fluid was allowed to thaw completely at temperatures not exceeding $37^{\circ} \mathrm{C}$. A segment of a silicon isolator containing three wells was attached onto each of the modified glass surfaces. Ten $\mu \mathrm{L}$ of synovial fluid were transferred into each well using sterile micro pipettes. Upon the addition of synovial fluid, the wells are covered with a transparent polymer cover slip to avoid contamination by foreign particles.

\section{Preparation of silver colloids}

Silver colloids were prepared according to the following procure: a $98 \mathrm{~mL}$ aqueous solution of 6.5 $\mathrm{mM}$ of $\mathrm{AgNO}_{3}$ was heated in a round bottom flask equipped with a condenser to $>80^{\circ} \mathrm{C}$ of while stirring. Two $\mathrm{mL}$ of $11.6 \mathrm{mM}$ trisodium citrate solution was added dropwise to silver nitrate solution. The temperature of the mixture was kept elevated for 10 minutes and then was reduced to room temperature.

\section{Uric acid re-crystallization}

The initial study entailed the dissolution of uric acid in deionized water at reported concentrations corresponding to elevated plasma urate levels, at approximately body temperature: $8 \mathrm{mg}$ of uric acid crystals were added to $20 \mathrm{~mL}$ of deionized water and stirred continuously at $37^{\circ} \mathrm{C}$ until completely dissolved. $30 \mu \mathrm{L}$ of uric acid solution were added to each well on the collagen modified glass slides and allowed to recrystallize at room temperature for 1 hour or until no further change was observed.

\section{De-crystallization of uric acid in synovial fluid on collagen-modified glass slides}

De-crystallization of uric acid was monitored under different experimental conditions. In this regard, 10 $\mu \mathrm{L}$ of gold or silver colloid solution were mixed in to each well that already contain $10 \mu \mathrm{L}$ of synovial fluid, and the slides observed using an optical microscope at room temperature or alternately heated in the commercially available microwave oven (60 seconds on and 30 seconds off: microwave power level 1 or duty cycle of 3 seconds; $900 \mathrm{~W}$ maximum power, Frigidaire Model No. FCM09Z03KB) observed at 1 minute intervals (there was 30 second delay in between microwave heating steps to collect the images of uric acid crystals using an optical microscope stationed in close proximity to the microwave oven). The crystals at each interval were quantified and measured using Motic Software.

\section{Results and discussion}

Figure 1 shows the optical images of the uric acid crystals grown on collagen-modified glass slides at room temperature (labeled as $\mathrm{t}=0 \mathrm{~min}$ ) and after exposure to three different experimental conditions (i.e., treatments) for $\mathrm{t}=10 \mathrm{~min}$ : i). no colloids, a control experiment, ii) gold colloids and iii) silver colloids with microwave heating and room temperature (no microwave heating). Figure 1(a) shows that none of the three treatments of uric acid crystals at room temperature for $10 \mathrm{~min}$ resulted in any notable change in the size or the number of uric acid crystals. The use of low power microwave heating of the uric acid crystals in the absence of colloids was also ineffective in reducing the number and size of uric acid crystals. Alternatively, significant reduction in the number of uric acid crystals was observed within 10 minutes of treatment with gold colloids and low power microwave irradiation (Fig. 1(b)). No significant change in the size and number of uric acid crystals was observed upon treatment of uric acid crystals with silver colloids and low power microwave heating (Fig. 1(b)).

To determine the minimal effective time of each 


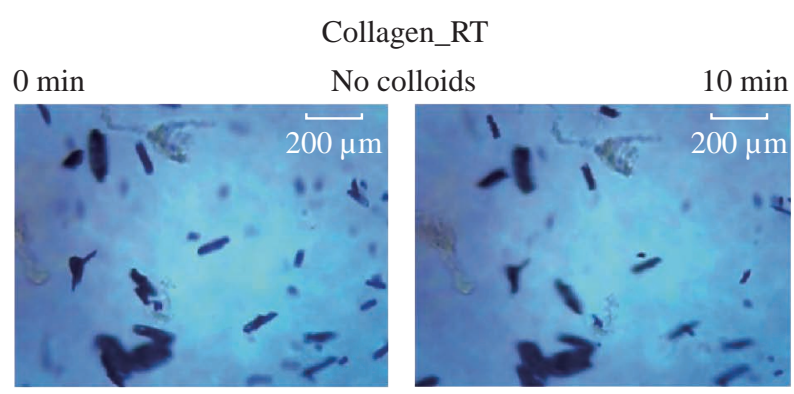

Gold colloids
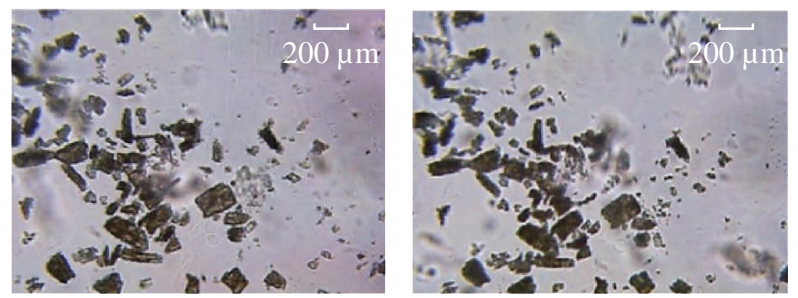

Silver colloids
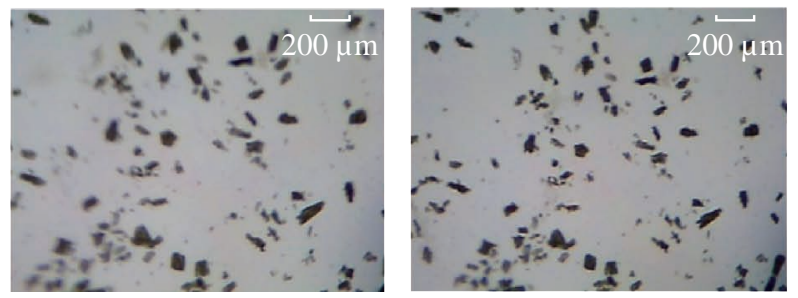

(a)

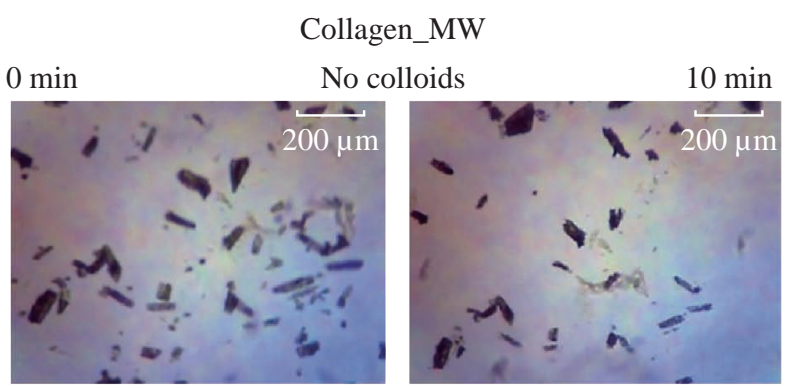

Gold colloids

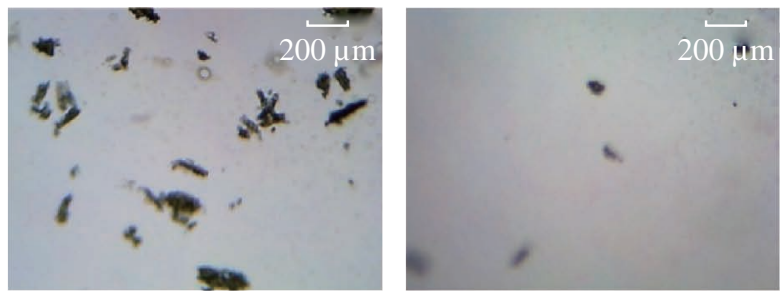

Silver colloids
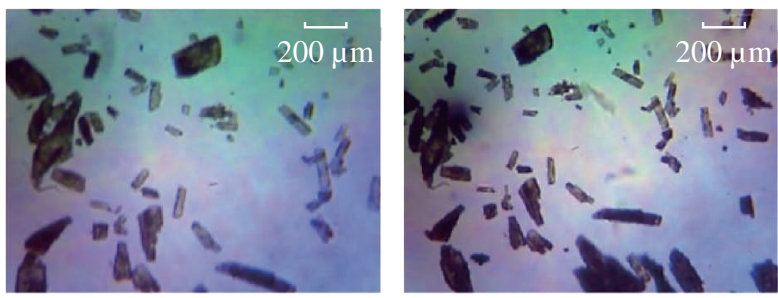

(b)

Fig. 1 (a) Initial (1 min) and (b) final (after $10 \mathrm{~min}$ ) microscope images of uric acid crystals incubated at room temperature (RT) and using microwave heating (MW) in the absence of colloids and in the presence gold and silver colloids. Please note microwave heating was turned on for 60 seconds and turned off 30 seconds to collect these images. Microwave power level 1 or duty cycle of 3 seconds used with $900 \mathrm{~W}$ microwave source).

treatment and to monitor the changes in crystal morphology with respect to the time of microwave heating, optical images of uric acid crystals for each treatment were captured at 1 minute intervals for the entire 10 min duration of study for each experiment, and is shown in Figs. 2 and 3, (S1, S2 and S3 Supporting Information). Figure 2 shows the timed microscope images of uric acid crystals incubated at room temperature for $10 \mathrm{~min}$ in the presence of gold colloids. Despite the presence of the gold colloids, there is no apparent change in the number or the size of crystals after $10 \mathrm{~min}$ of treatment. The addition of gold colloids, while proven to be ineffective at room temperature, resulted in significant decrease in the number and the size of uric acid crystals when subjected to intermittent low level microwave heating as depicted in Fig. 3. The success of the decrystallization is assessed based on the reduction of the total number of crystals at each time interval relative to that at $\mathrm{t}=0 \mathrm{~min}$ of the treatment. For example, there is a slight decrease in the number of crystals with the remaining crystals appear smaller than in the initial image 2 minutes after the addition of the gold colloids. Approximately $50 \%$ of the uric acid crystals were decrystallized within 3 minutes of low power microwave heating in the presence of gold colloids. Continuation of microwave heating of the samples up to $10 \mathrm{~min}$ further reduced the number of uric acid crystals to $10 \%$ of the initial number of uric acid crystals. Figures S1 and S2 (Supporting Information) show that the use of silver colloids or the absence of colloids at room temperature or microwave heating have no effect on the size and the number of uric acid crystals. These observations demonstrate the effectiveness of the combined use of microwave heating with gold colloids in de-crystallization of uric acid crystals in synovial fluid on collagen-modified glass slides.

Figure 4 summarizes the effectiveness of each treatment, as assessed by the retention rate of uric acid crystals. In this regard, the crystal percentage retention was calculated by dividing the total number of uric acid crystals at any time of the experiment counted from the optical microscope images by the initial number of uric acid crystals. The calculated crystal retention 
Collagen_RT_Gold colloids
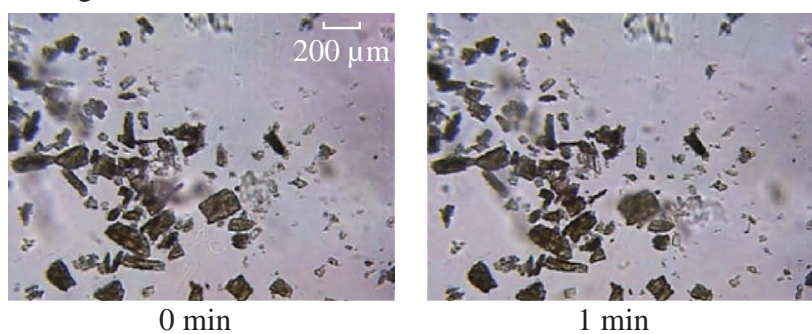

$1 \mathrm{~min}$
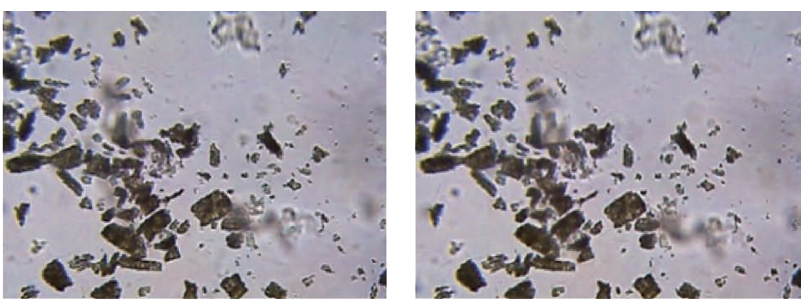

$2 \mathrm{~min}$
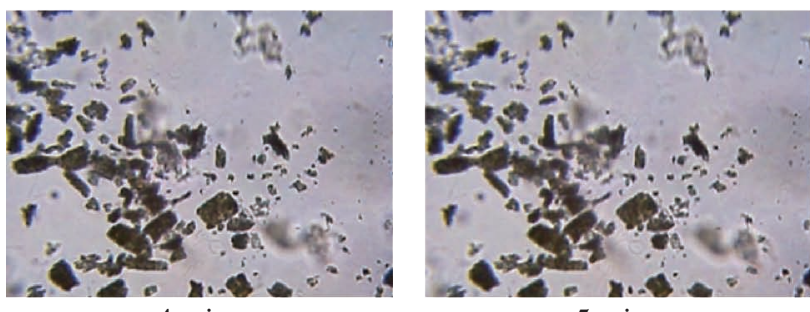

$5 \mathrm{~min}$

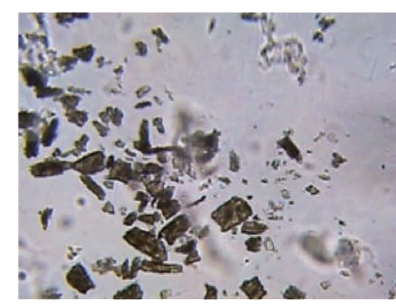

$6 \mathrm{~min}$
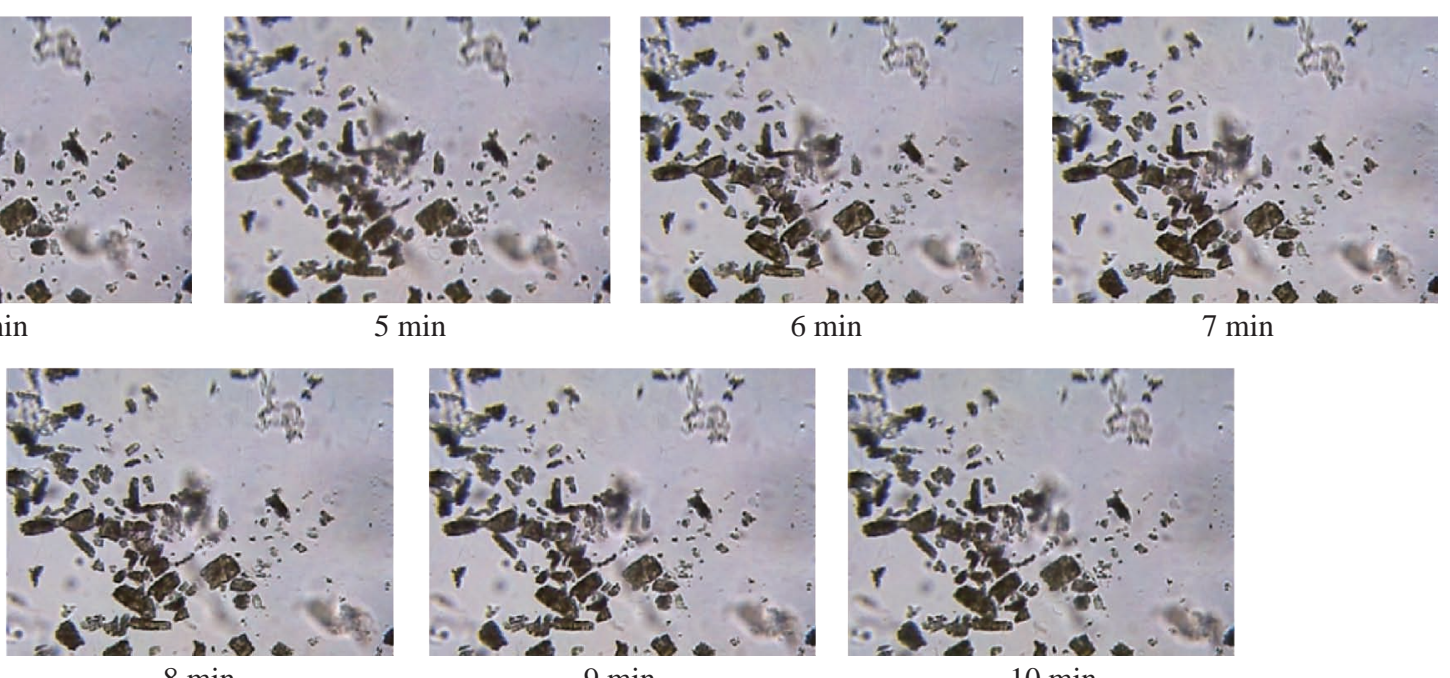

Fig. 2 Timed microscope images of uric acid crystals incubated at room temperature for 10 minutes in the presence of gold colloids.

Collagen_MW_Gold colloids

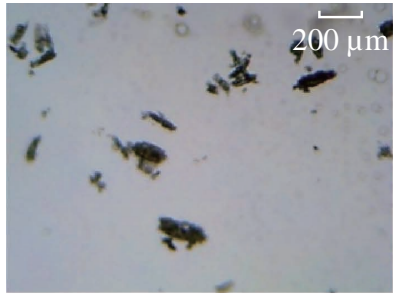

0 min

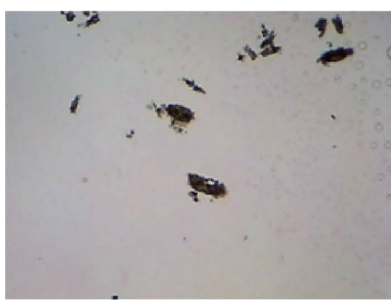

$4 \min$

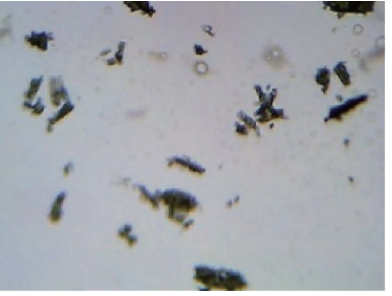

1 min

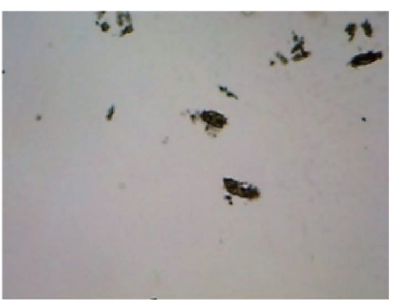

5 min

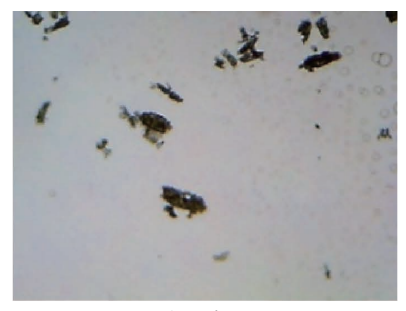

2 min

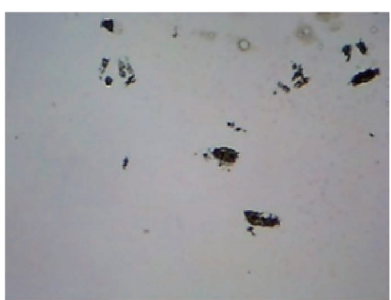

$6 \min$

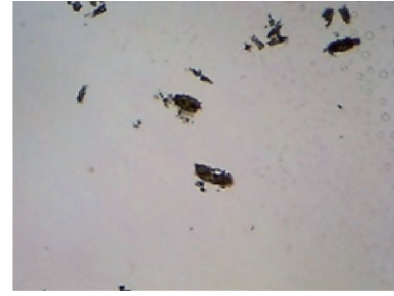

$3 \min$

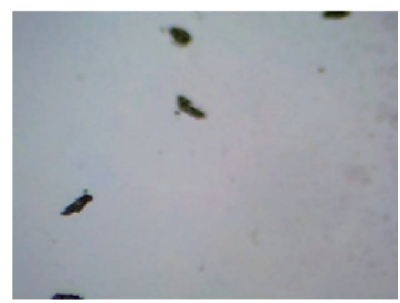

$7 \mathrm{~min}$

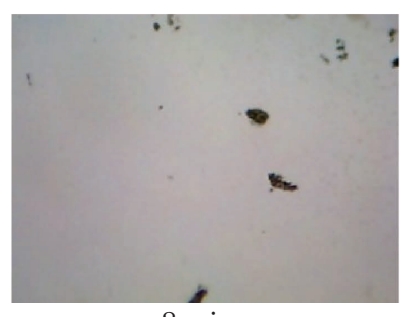

$8 \mathrm{~min}$

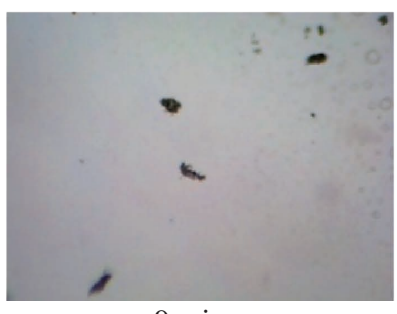

9 min

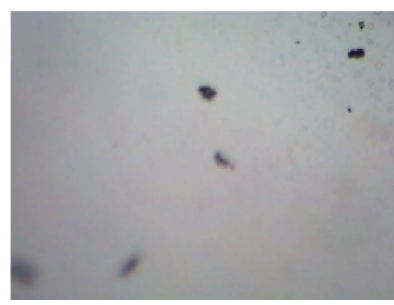

$10 \min$

Fig. 3 Timed microscope images of uric acid crystals exposed to microwave heating (MW, power level 1) for 10 minutes in the presence of gold colloids. 
Collagen_RT_Silver colloids
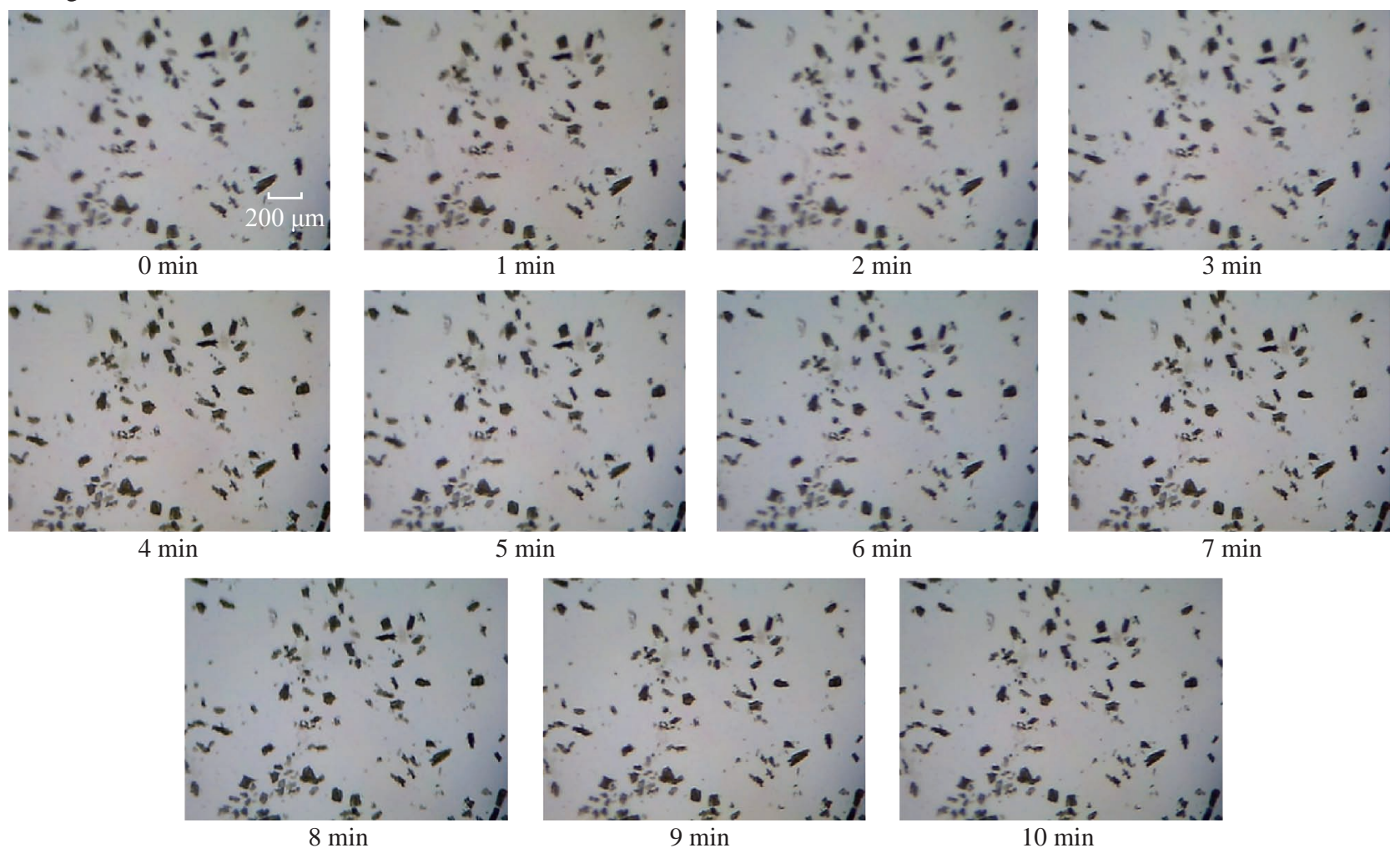

Fig. S1 Timed microscope images of uric acid crystals incubated at room temperature for 10 minutes in the presence of silver colloids.

Collagen_MW_Silver colloids

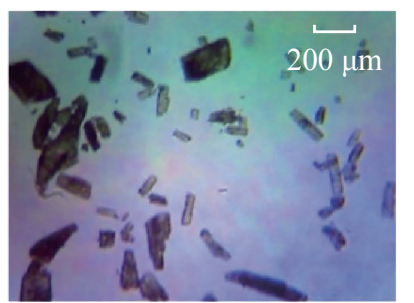

0 min

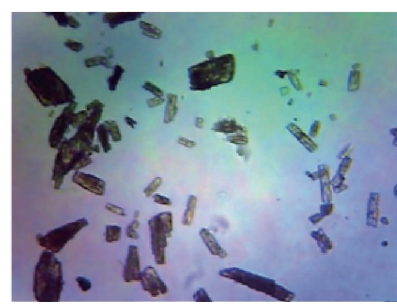

4 min

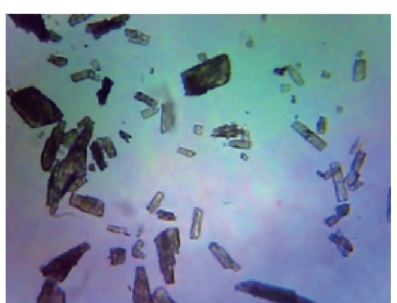

1 min

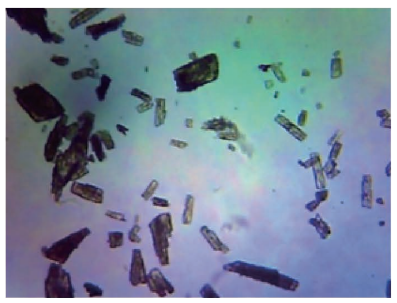

5 min

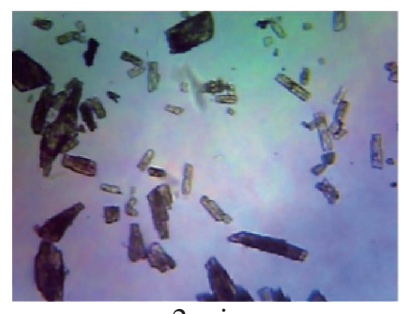

2 min

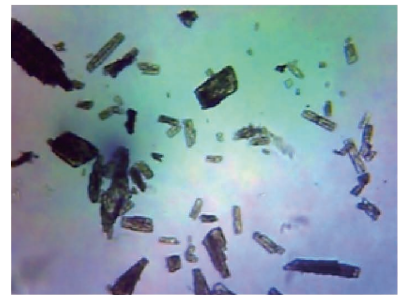

6 min

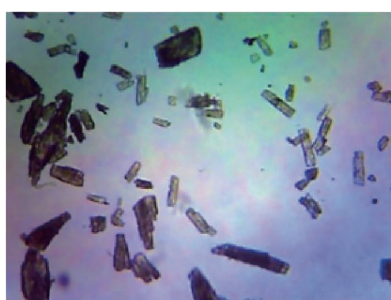

$3 \mathrm{~min}$

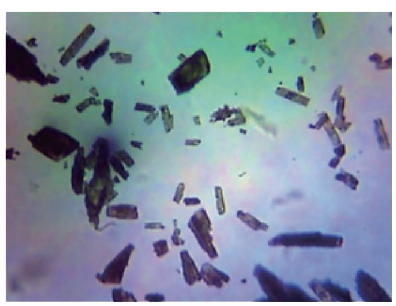

$7 \mathrm{~min}$

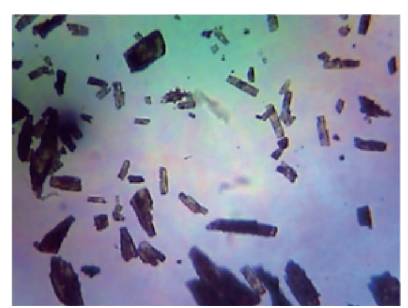

8 min

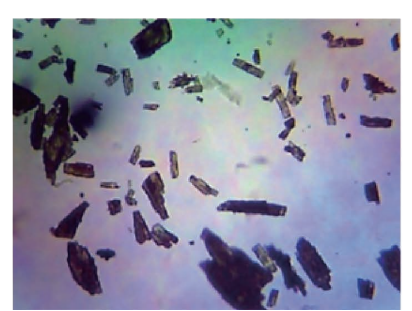

9 min

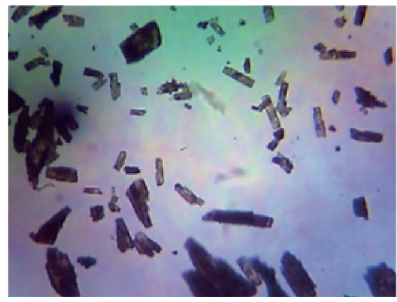

$10 \mathrm{~min}$

Fig. S2 Timed microscope images of uric acid crystals exposed to microwave heating (MW, power level 1) for 10 minutes in the presence of silver colloids. 


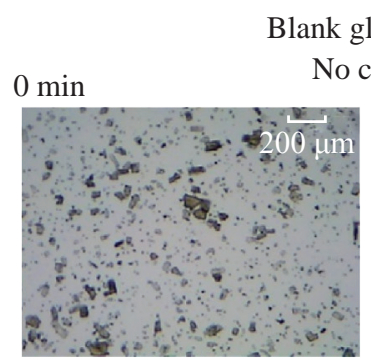

No colloids

Gold colloids
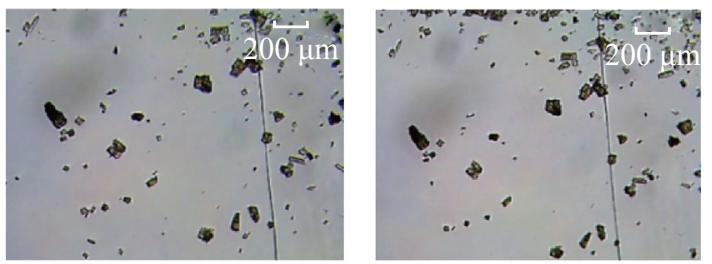

Silver colloids
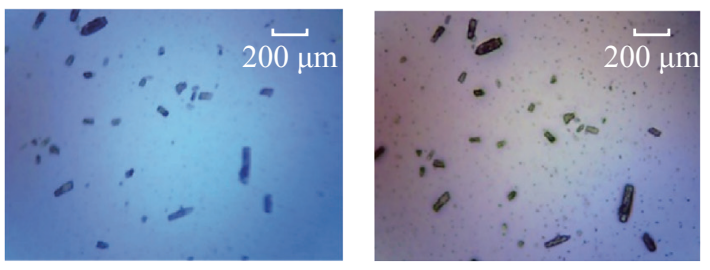

(a)
Blank glass_MW

No colloids
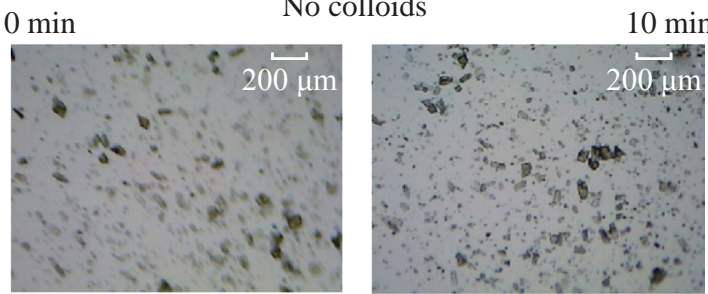

Gold colloids
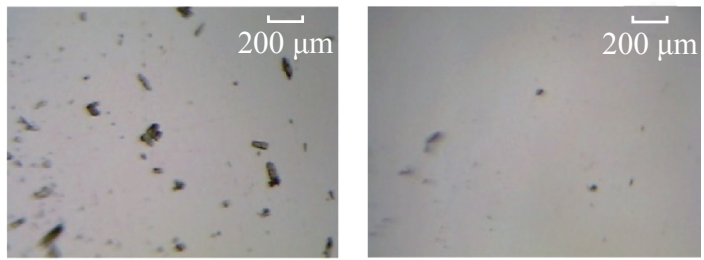

Silver colloids
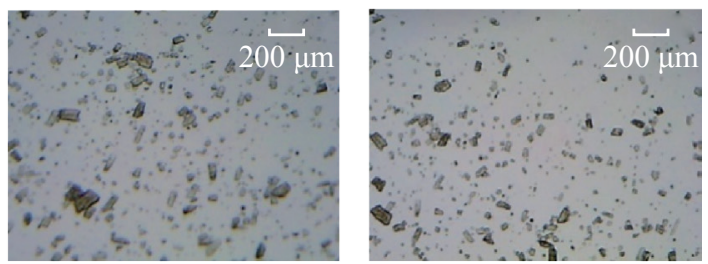

(b)

Fig. S3 (a) Initial (1 min) and (b) final (after $10 \mathrm{~min}$ ) microscope images of uric acid crystals incubated at room temperature (RT) and using microwave heating (MW) in the absence of colloids and in the presence gold and silver colloids.
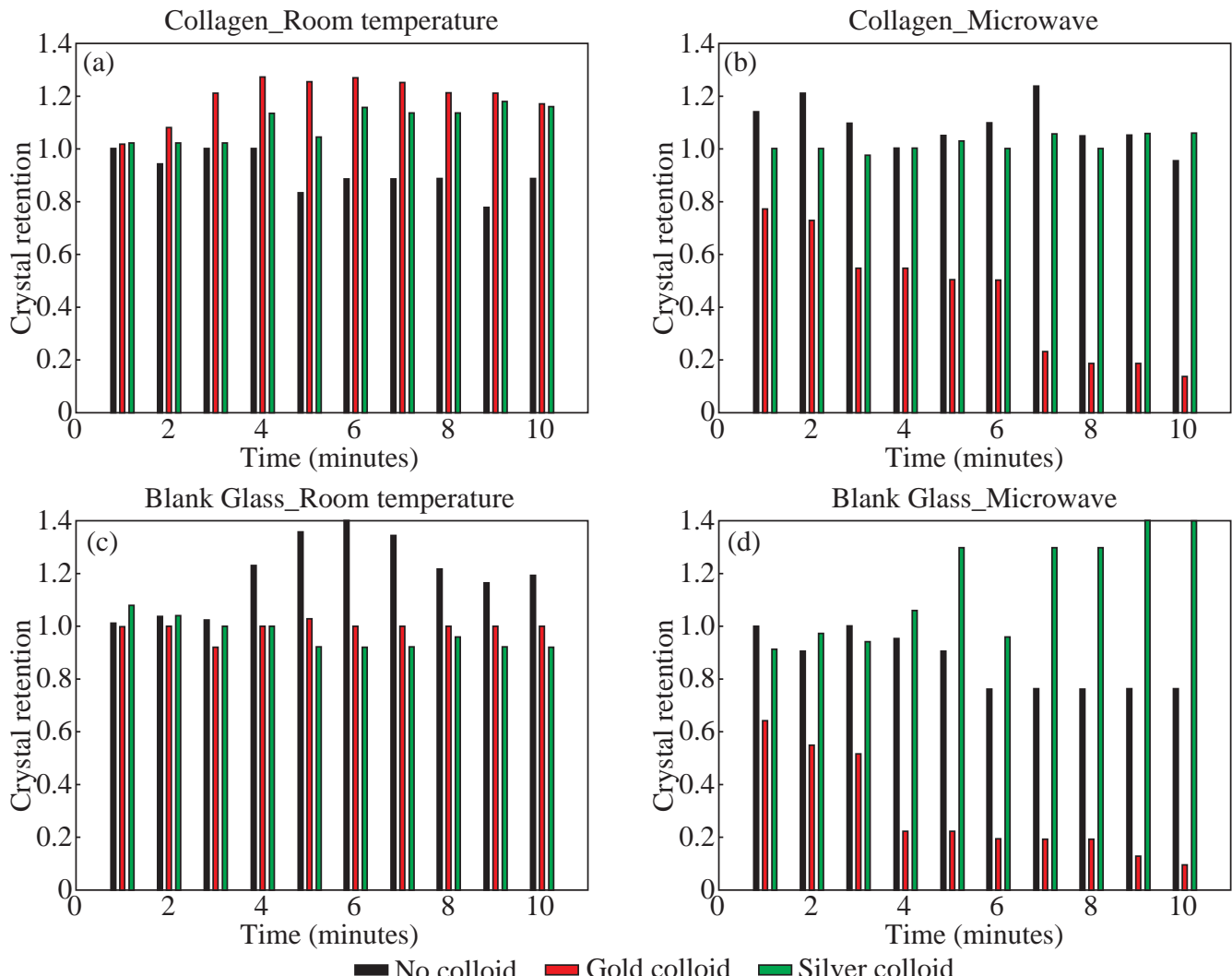

Fig. 4 Calculated retention rate of uric acid crystals on collagen-modified glass slides (a) at room temperature and (b) using microwave heating and (c) on a blank glass surface (i.e., no collagen films, a control surface) at room temperature and (d) using microwave heating. 
value at $\mathrm{t}=0 \mathrm{~min}$ is 1.0 and decreases $<1.0$ when decrystallization occur. Retention values $>1.0$ implies the appearance of additional crystals that can be grown from solution. Figure 4(a) shows that the addition of gold or silver colloids resulted in an increase in the retention rate, which implies the presence of additional uric acid crystals grown from solution in the presence of gold and silver colloids. The retention rate of uric acid crystals in the absence of colloids at room temperature showed a $10 \%$ decrease, which can be attributed to the dissolution of uric acid crystals. Figure 4(b) shows that microwave heating of uric acid crystals in the gold colloids resulted in the reduction of the crystal retention rate by $30 \%$ after 3 min and $80 \%$ after $10 \mathrm{~min}$. On the other hand, microwave heating of uric acid crystals in the presence of silver colloids or the absence of colloids did not result in a significant change in the crystal percentage retention.

The experiments described above were also repeated on blank glass slides (i.e., no collagen, Fig. 4(c), 4(d), S3, S4 and S5) to demonstrate the case of gout disease when the collagen is completely depleted in human bones. Similar to the results observed on collagenmodified glass slides, the presence of silver colloids and gold colloids at room temperature did not result in the change in the number of uric acid crystals. A slight increase in the number of uric acid crystals was observed when both silver and gold colloids were omitted from the blank glass surfaces, which indicates the further growth of uric acid crystals.

It is also important to comment on the change in the crystal size distribution with respect to the number of uric acid crystals in all treatments used in this study. Figure S6 (Supporting Information) that there is no significant change in the uric acid crystal size distribution in silver colloids and no colloids treatments carried out at room temperature. However, the use of gold colloids resulted in the growth of uric acid crystals with size of $<25 \mathrm{~mm}$, which can be attributed to the previous observation that colloids can serve as nucleation sites for the growth of biological crystals at room temperature [20, 21]. Figure S7 (Supporting Information) shows that the size of the uric acid crystals were up to $125 \mathrm{~mm}$ before the commencement of the treatment with gold colloids and microwave heating (Line II at $\mathrm{t}=0 \mathrm{~min}$, initial number of uric acid crystals was 22). As the microwave heating is applied and continued for $10 \mathrm{~min}$, the size distribution of uric acid crystals narrowed down to $<50 \mathrm{~mm}$ and the total number of uric crystals decreased to 4 . The use of microwave heating with silver colloids or no colloids did not result in a significant change in the size

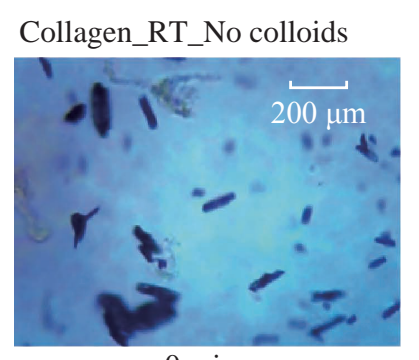

$0 \min$

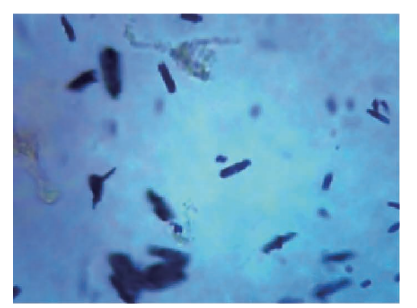

$4 \min$

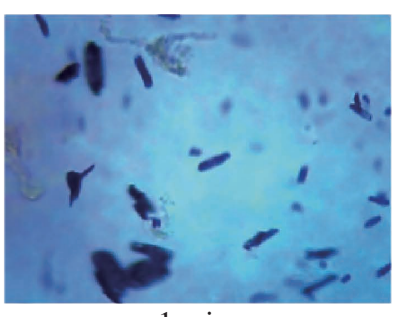

$1 \min$

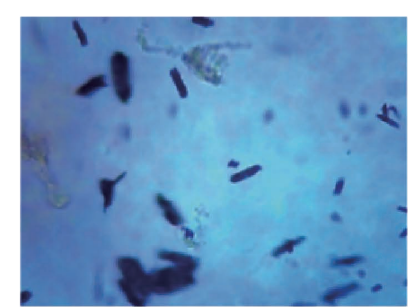

$5 \min$

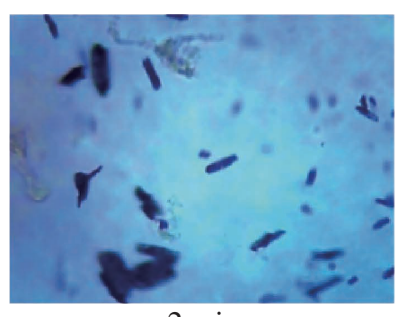

$2 \min$

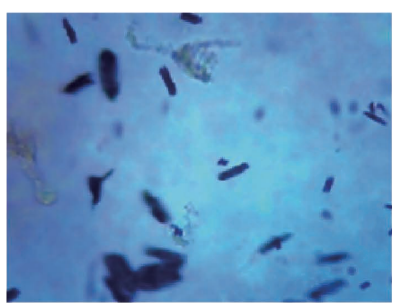

$6 \mathrm{~min}$

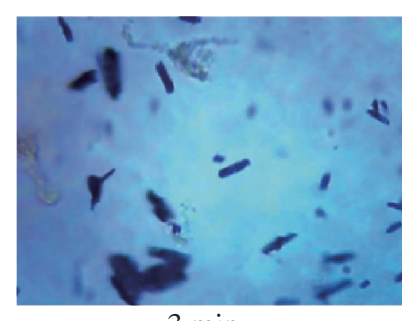

3 min

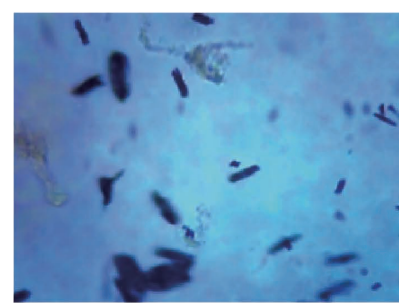

$7 \mathrm{~min}$

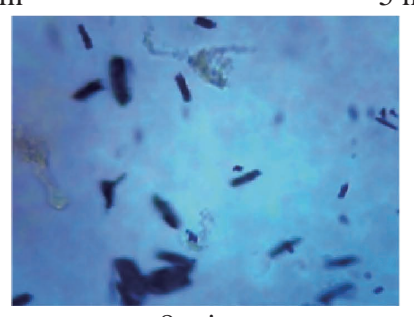

$8 \mathrm{~min}$

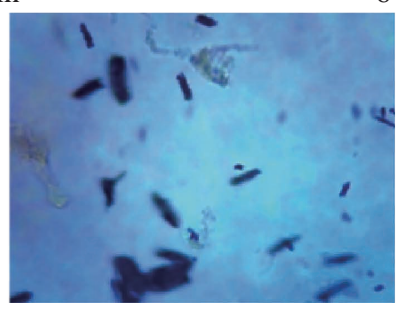

$9 \min$

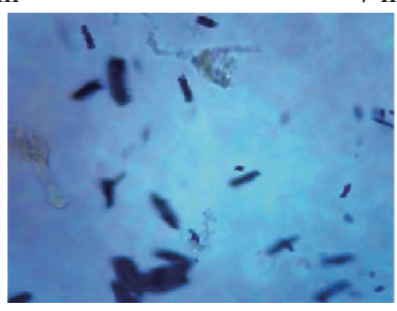

$10 \mathrm{~min}$

Fig. S4 Timed microscope images of uric acid crystals incubated at room temperature for 10 minutes in the absence of colloids. 
Collagen_MW_No colloids

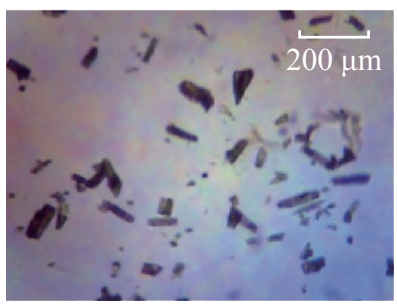

$0 \mathrm{~min}$

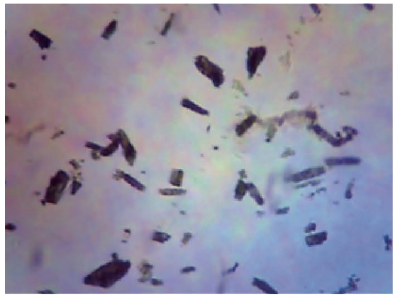

$4 \mathrm{~min}$

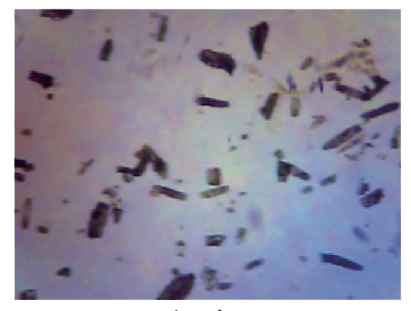

1 min

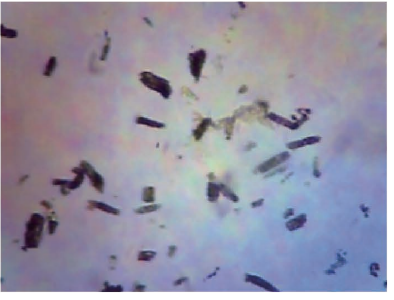

5 min

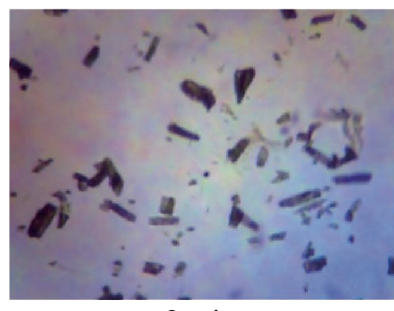

$2 \min$

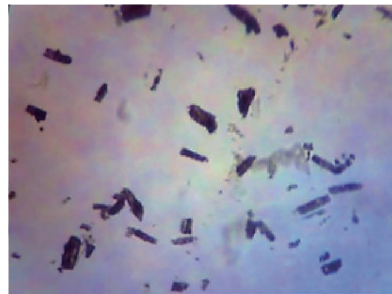

6 min

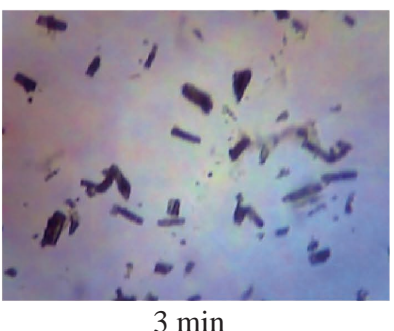

$3 \mathrm{~min}$

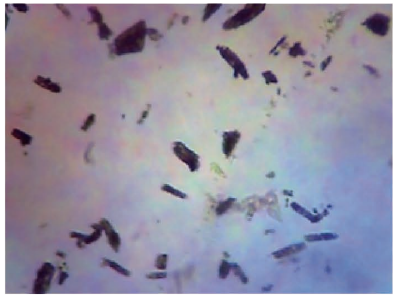

$7 \mathrm{~min}$

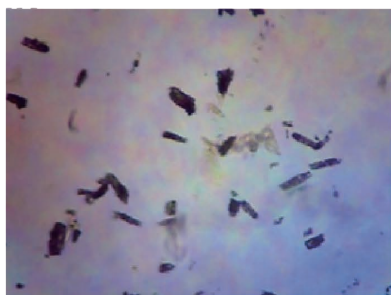

$8 \min$

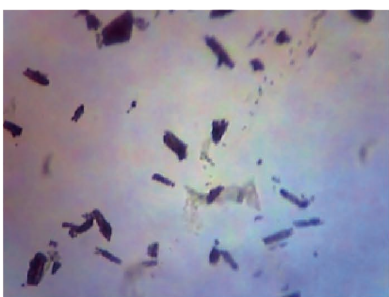

$9 \min$

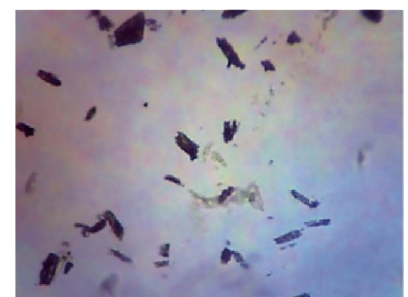

$10 \min$

Fig. S5 Timed microscope images of uric acid crystals exposed to microwave heating (MW, power level 1) for 10 minutes in the absence of colloids.

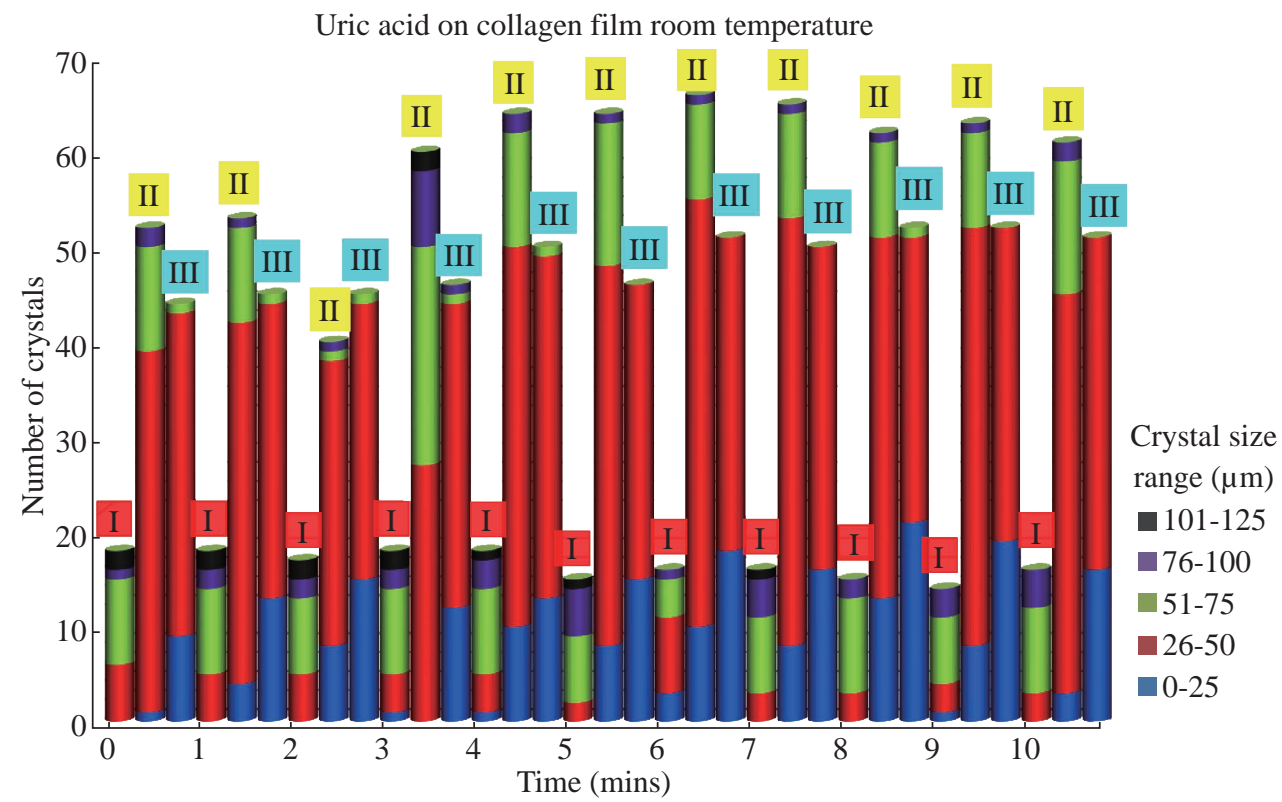

Fig. S6 Change in the number of uric acid crystals at room temperature in the presence of no colloids (I), gold colloids (II) and silver colloids (III) and the corresponding size ranges present for each experiment.

distribution and the number of uric acid crystals, which implies that these treatments are in effective for the decrystallization of uric acid crystals.

To understand the observed differences between gold and silver colloids in terms of their ability to decrystallize uric acids crystals in the MAMAD technique, the optical absorbance spectra of these colloids in the presence of synovial fluid during 


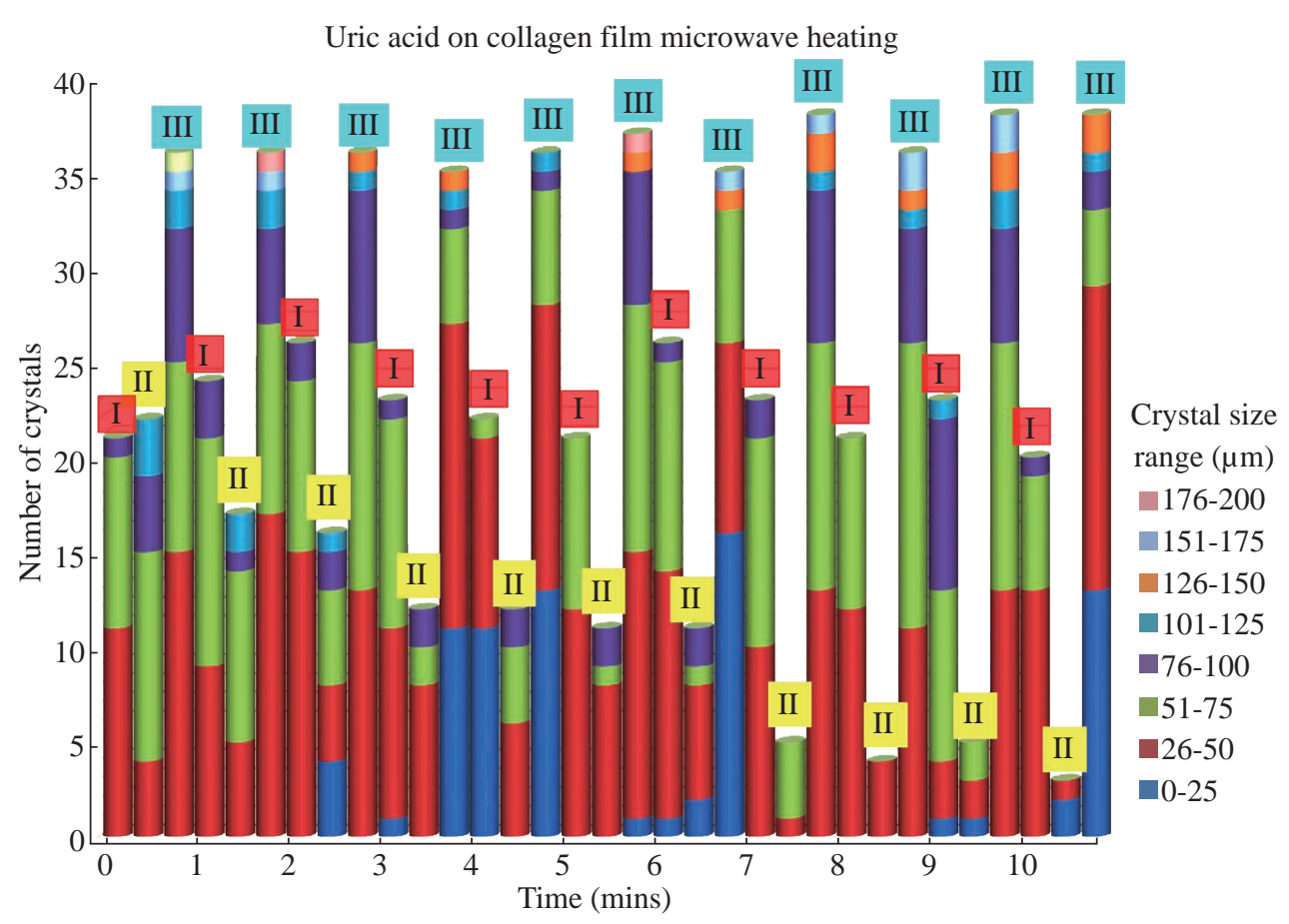

Fig. S7 Change in the number of uric acid crystals exposed to microwave heating in the presence of no colloids (I), gold colloids (II) and silver colloids (III) and the corresponding size ranges present for each experiment.

intermittent microwave heating were measured (Fig. 5). We note that the solutions containing metal colloids and synovial fluids were not allowed to dry out during microwave heating by using a plastic cover that is placed over the top of the solution. Figure 5(a) shows that gold colloids solution as purchased (labeled as Au in the figure) has a surface plasmon resonance (SPR) peak at $520 \mathrm{~nm}$ with narrow absorbance values at wavelengths $>600 \mathrm{~nm}$ and serves as the control sample to assess the stability of gold colloids in synovial fluid. The addition of synovial fluid to gold colloids (1:1 v:v, similar to the decrystallization experiments) result in a slight red shift in the SPR peak (to $532 \mathrm{~nm}$ ) due to the changes in dielectric constant of the solution and the appearance of an additional peak $410 \mathrm{~nm}$ that can be attributed to synovial fluid (labeled as $\mathrm{Au}+\mathrm{SF}$ at 0 min). As the gold colloid and synovial fluid mixture is exposed to intermittent microwave heating ( $1 \mathrm{~min}$ intervals), the wavelength of the SPR peak of the gold colloids at $532 \mathrm{~nm}$ did not change. However, a broadening in the absorption spectra of the gold colloids at wavelengths $>600 \mathrm{~nm}$ was observed. These observations imply that although the gold colloids remained as individual particles, the inter-particle distance was decreased during intermittent microwave heating in the presence of synovial fluid.

It is important to note that the observed stability of gold colloids in synovial fluid explains why gold colloids is efficient in the reduction of uric acid crystals using the MAMAD technique. On the other hand, unlike gold colloids, silver colloids aggregated out of the solution immediately after the addition of synovial fluid due to the lack of protective citrate around the silver colloids and remained aggregated during intermittent microwave heating. The lack of stability of silver colloids in synovial fluid explains the observations of ineffective decrystallization of uric acid crystals using silver colloids in the MAMAD technique. Moreover, the observed additional growth of uric acid crystals on surfaces in the presence of silver colloids (Fig. 4) can also be explained by the presence of silver colloids on the surface due to aggregation: silver colloids immobilized on to surfaces enhance the growth biological crystals, as we have reported previously [22, 23]. We note that the use of metal colloids in solution in conjunction with microwave heating is crux of the MAMAD technique for the decrystallization of crystals.

Since microwave heating is involved in the decrystallization of uric acid crystals presented in this study, it is important to investigate the changes in temperature of the system (Fig. 6(a)), which is comprised of solution where the gold colloids are present in synovial fluid and the collagen-modified glass slide surface where uric acid crystal are grown. Figure 6(b) shows the real-time temperature of 

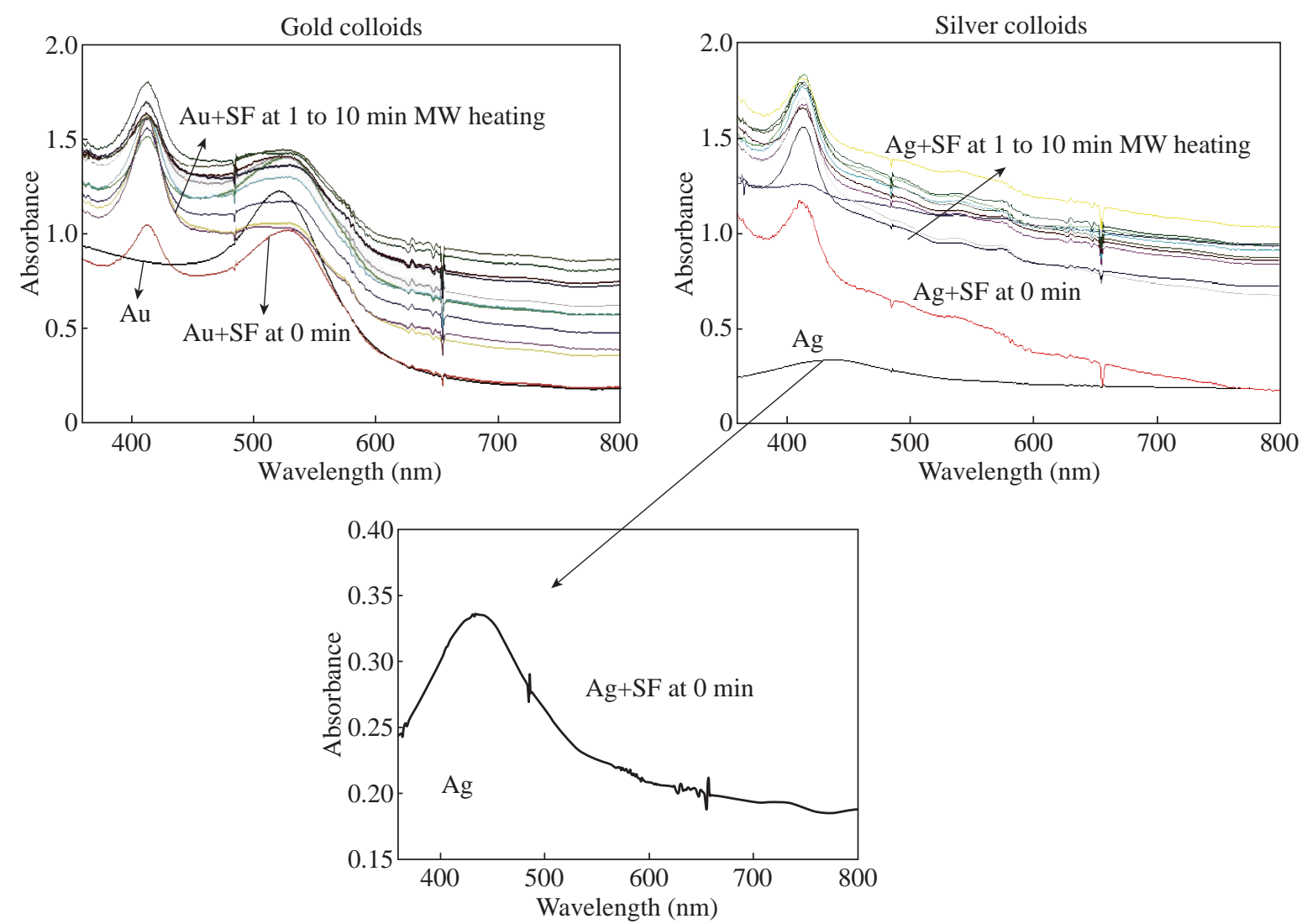

Fig. 5 Optical absorption spectrum of (a) gold colloids and (b) silver colloids in the absence and presence of synovial fluid (SF) during intermittent microwave heating (MW) at $1 \mathrm{~min}$ intervals for $10 \mathrm{~min}$. Inset- absorption spectrum of silver colloids in buffer.
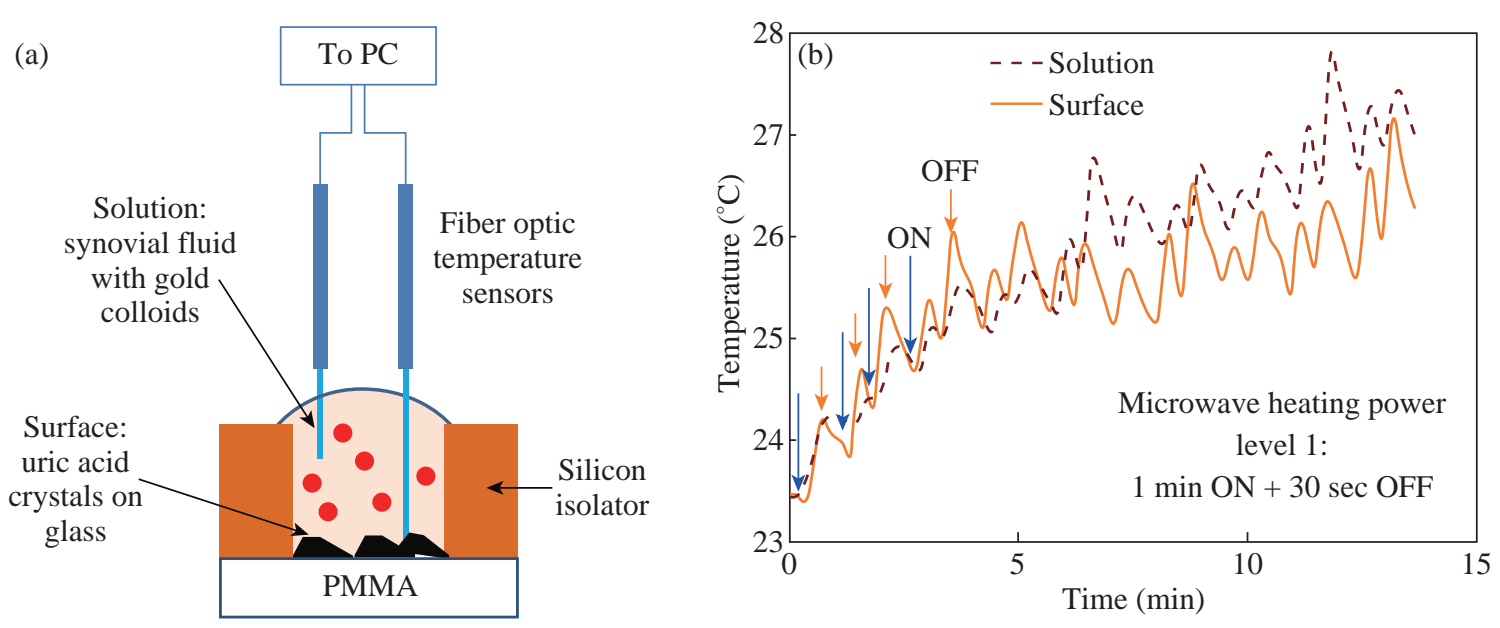

Fig. 6 (a) Real-time temperature of the solution and the surface of the glass slides in a well measured inside a $900 \mathrm{~W}$ microwave cavity using fiber optic temperature sensors. (b) Schematic depiction of the experimental setup for temperature measurements inside the microwave cavity.

the system during intermittent heating in a $900 \mathrm{~W}$ microwave cavity for $10 \mathrm{~min}$. The temperature of the surface and the solution was increased from $23.5^{\circ} \mathrm{C}$ to 26 and $26.5^{\circ} \mathrm{C}$, respectively, which demonstrates that a potential application of microwave heating with gold colloids will not cause significant increase in temperature.
It is also important to comment on the combined use of microwave heating and metal colloids in solution for effective de-crystallization of uric acid crystals in synovial fluid on collagen-modified glass slides. The unique feature of the combined use of gold colloids in solution and microwave heating for the potential treatment of gout is the reflection of electromagnetic 
energy and subsequent conversion to additional kinetic energy by gold colloids exposed to microwave irradiation without significant increase in the overall temperature of the system (Fig. 6(b)) [24, 25]. The increase in kinetic energy of gold colloids in solution placed in close proximity to uric acid crystals results in the collision of metal nanoparticles with uric acid crystals. Since the number of metal nanoparticles $\left(1 \times 10^{11}\right.$ particles $\left./ \mathrm{mL}\right)$ is significantly larger than the number of uric acid crystals (which are also 1000-fold larger in size than metal nanoparticles of $\sim 20 \mathrm{~nm}$ in diameter), these collisions result in the fracturing and eventual dissolution of the uric acid crystals (i.e., via Ostwald ripening). The solubility of uric acid crystals is increased due to the elevated stress-levels of the molecules on the solute-solvent interface of uric acid crystalline faces. The stress on these molecules can be elevated (by the collisions between the uric acid crystals and the gold colloids) due to their lack of coordination, which results in an overall increase in forces acting on the crystal lattice. Beyond a critical cluster size, there is a zero net force within a stable crystal owing to the balance between the surface tension directed inward and the electrical tension directed outward. The crystal easily fractures when a critical cluster size is not retained. An increase in solvent temperature also contributes to the increased solubility of uric acid crystals when exposed to microwave irradiation. As shown in Fig. 6, there is a $\sim 3{ }^{\circ} \mathrm{C}$ increase in the solvent temperature caused by the absorption of energy by the solvent molecules themselves. This increase in solvent temperature allows for the increased solubility of the uric acid and gradual reduction in size of the uric acid crystals. It is important to note that silver colloids and other metal colloids in solution can be used with the MAMAD technique by preventing their aggregation, hence the name MAMAD is appropriate for the technique. Our laboratory is currently working on the improvement of the model bone by using a 3-D printer technology and using a model synthetic human tissue. These results are being collected and will be reported in due course.

\section{Supporting information}

Additional information related to the each treatment of the uric acid crystals, and graphical depictions of the effects thereof on both number and size of crystals on both control and biological platforms is provided.

\section{Characterization of glass surfaces}

To simulate the de-crystallization of uric acid in biological medium in the joint of a bone, collagenmodified glass slide were employed. To confirm the progressive modification of the glass surface, the contact angle of a droplet of water was measured: $26.2^{\circ}$ on plain glass slides, $85.5^{\circ}$ on the silanized surface and $40.3^{\circ}$ on the fully modified collagen thin film. The observed increase in the contact angle $\left(26.2^{\circ}\right.$ to $\left.85.5^{\circ}\right)$ indicates that silanization of glass slides increases the hydrophobicity of the glass surface and the subsequent decrease in the contact angle (from $85.5^{\circ}$ to $40.3^{\circ}$ ) proves that collagen is attached to the silanized glass slides.

Table S1 Contact angles of a water droplet marking the progressive modification of the glass slides with HTMS and collagen films

\begin{tabular}{ccc}
\hline Platform & $\begin{array}{c}\text { Water contact } \\
\text { angle (degrees) }\end{array}$ & Images \\
\hline Blank glass slide & $26.2 \pm 1.18$ \\
\hline $\begin{array}{c}\text { Silanized glass slide } \\
\begin{array}{c}\text { Silanized glass slide } \\
\text { + Collagen }\end{array}\end{array}$ & $40.5 \pm 5.03$ \\
\hline
\end{tabular}

\section{References}

[1] M.B. Davidson, S. Thakkar, J.K. Hix, et al., Pathophysiology, clinical consequences, and treatment of tumor lysis syndrome. The American Journal of Medicine, 2004, 116: 546-554.

[2] J. Seegmiller, A.I. Grayzel, L. Laster, et al., Uric acid production in gout. Journal of Clinical Investigation, 1961, 40: 1304.

[3] H.K. Choi, S. Liu, G. Curhan, Intake of purine-rich foods, protein, and dairy products and relationship to serum levels of uric acid: The Third National Health and Nutrition Examination Survey. Arthritis \& Rheumatism, 2005, 52: 283-289.

[4] Y. Zhu, B.J. Pandya, H.K. Choi, Prevalence of gout and hyperuricemia in the US general population: The National Health and Nutrition Examination Survey 2007-2008. Arthritis \& Rheumatism, 2011, 63: 3136-3141.

[5] H.K. Choi, D.B. Mount, A.M. Reginato, Pathogenesis of gout. Annals of Internal Medicine, 2005, 143: 499-516.

[6] N. Dalbeth, B. Pool, G.D. Gamble, et al., Cellular characterization of the gouty tophus: A quantitative analysis. Arthritis \& Rheumatism, 2010, 62: 1549-1556.

[7] F. Martinon, V. Petrilli, A. Mayor, et al., Gout-associated uric acid crystals activate the NALP3 inflammasome. 
Nature, 2006, 440: 237-241.

[8] K.Y. Kim, H. Ralph Schumacher, E. Hunsche, et al., A literature review of the epidemiology and treatment of acute gout. Clinical Therapeutics, 2003, 25: 1593-1617.

[9] S.P. Juraschek, L.C. Kovell, E.R. Miller, 3rd, et al., Gout, urate-lowering therapy, and uric acid levels among adults in the United States. Arthritis Care Res (Hoboken), 2015, 67: 588-592.

[10] A.L. Weaver, Epidemiology of gout. Cleveland Clinic Journal of Medicine, 2008, 75: S9-S12.

[11] J. Page, D. Henry, Consumption of nsaids and the development of congestive heart failure in elderly patients: An underrecognized public health problem. Archives of Internal Medicine, 2000, 160: 777-784.

[12] S.C. Liu, L. Xia, J. Zhang, et al., Gout and Risk of Myocardial Infarction: A Systematic Review and MetaAnalysis of Cohort Studies. PLoS One, 2015, 10: e0134088.

[13] R.A. Terkeltaub, D.E. Furst, K. Bennett, et al., High versus low dosing of oral colchicine for early acute gout flare: Twenty-four-hour outcome of the first multicenter, randomized, double-blind, placebo-controlled, parallelgroup, dose-comparison colchicine study. Arthritis \& Rheumatism, 2010, 62: 1060-1068.

[14] A.S. Kesselheim, D.H. Solomon, Incentives for Drug Development - The Curious Case of Colchicine. New England Journal of Medicine, 2010, 362: 2045-2047.

[15] B. Kioko, T. Ogundolie, M. Adebiyi, et al., Decrystallization of Uric Acid Crystals in Synovial Fluid Using Gold Colloids and Microwave Heating. Nano Biomed Eng., 2014, 6: 104-110.

[16] C.J. Murphy, A.M. Gole, J.W. Stone, et al., Gold Nanoparticles in Biology: Beyond Toxicity to Cellular Imaging. Accounts of Chemical Research, 2008, 41: 17211730.

[17] A.V. Liopo, A. Conjusteau, M. Konopleva, et al., Laser nanothermolysis of human leukemia cells using functionalized plasmonic nanoparticles. Nano Biomed Eng., 2012, 4: 66-75.

[18] I. Bisson, M. Kosinski, S. Ruault, et al., Acrylic acid grafting and collagen immobilization on poly(ethylene terephthalate) surfaces for adherence and growth of human bladder smooth muscle cells. Biomaterials, 2002,
23: 3149-3158.

[19] A. Revzin, R.G. Tompkins, M. Toner, Surface Engineering with Poly(ethylene glycol) Photolithography to Create High-Density Cell Arrays on Glass. Langmuir, 2003, 19: 9855-9862.

[20] A.M. Alabanza, M. Mohammed, K. Aslan, Crystallization of Amino Acids on a 21-well Circular PMMA Platform using Metal-Assisted and Microwave-Accelerated Evaporative Crystallization. Nano Biomed Eng, 2013, 5: 140-147.

[21] T.A. Grell, M.A. Pinard, D. Pettis, et al., Rapid crystallization of glycine using metal-assisted and microwave-accelerated evaporative crystallization: the effect of engineered surfaces and sample volume. Nano Biomed Eng, 2012, 4: 125-131.

[22] A.M. Alabanza, M. Mohammed, K. Aslan, Crystallization of L-alanine in the Presence of Additives on a Circular PMMA Platform Designed for Metal-Assisted and Microwave-Accelerated Evaporative Crystallization. Cryst Eng Comm, 2012, 14: 8421-8431.

[23] A.M. Alabanza, E. Pozharski, K. Aslan, Rapid Crystallization of l-Alanine on Engineered Surfaces by Use of Metal-Assisted and Microwave-Accelerated Evaporative Crystallization. Crystal Growth \& Design, 2011, 12: 346-353.

[24] E. Araya, I. Olmedo, N. Bastus, et al., Gold Nanoparticles and Microwave Irradiation Inhibit Beta-Amyloid Amyloidogenesis. Nanoscale Research Letters, 2008, 3: 435-443.

[25] K. Aslan, C.D. Geddes, Microwave-accelerated ultrafast nanoparticle aggregation assays using gold colloids. Analytical chemistry, 2007, 79: 2131-2136.

Copyright $₫ 2015$ Yehnara S.B. Ettinoffe, Bridgit M. Kioko, Brittney I. Gordon, Nishone A. Thompson, Morenike Adebiyi, Kevin Mauge-Lewis, Taiwo O. Ogundolie, Enock Bonyi, Muzaffer Mohammed and Kadir Aslan. This is an open-access article distributed under the terms of the Creative Commons Attribution License, which permits unrestricted use, distribution, and reproduction in any medium, provided the original author and source are credited. 